\title{
Comparison of beliefs about cardiovascular medicines in Polish and American patients:
}

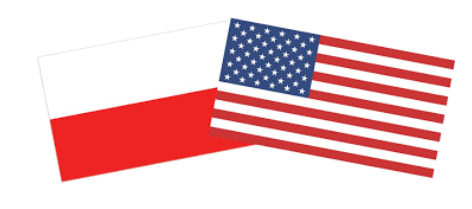

\section{Introduction} Patient adherence to medication is a constant concern and challenge
for healthcare. Patients varying beliefs about medicines impact how adherent they are to their medications; it has been seen that two of the most important factors are necessity for the given treatment and concerns about the treatment. Given this, the Necessity-Concern Framework (NCF) could help shape interventions to improve adherence and outcomes ${ }^{1}$. Also, cultural differences can play an important factor when evaluating these differences as different countries may have different medical systems and general beliefs. All of these components influence many aspects of medicine, including the doctor-patient relationship.

\section{Objectives}

The aim of this study was to compare the beliefs about patient's prescription medications in Lodz, Poland to those in Detroit, Michigan. The aim was to find any key differences between these groups to get a better understanding of the patient's perspective of their medications. The study specifically evaluates patients taking cardiovascular medication for common diseases such as hypertension, coronary artery disease, arrythmias and congestive heart failure.

\section{Materials and Methods} A paper questionnaire was given to general outpatient clinics,
pharmacies and senior living homes. The original English version ${ }^{2}$ as well as the recently validated Polish version of the Beliefs about Medicines Questionnaire (BMQ) ${ }^{3}$ was used together with the Adherence to Refills and Medications Scale (ARMS) ${ }^{4}$. Additionally, patients were asked to answer demographic questions regarding sex, age, place of residence and socioeconomic status.

\section{a cross sectionall study}

Scarlett Olejnik-Brzusek, Michał Seweryn Karbownik

Medical University of Lodz, Poland

Department of Pharmacology \& Toxicology
Results

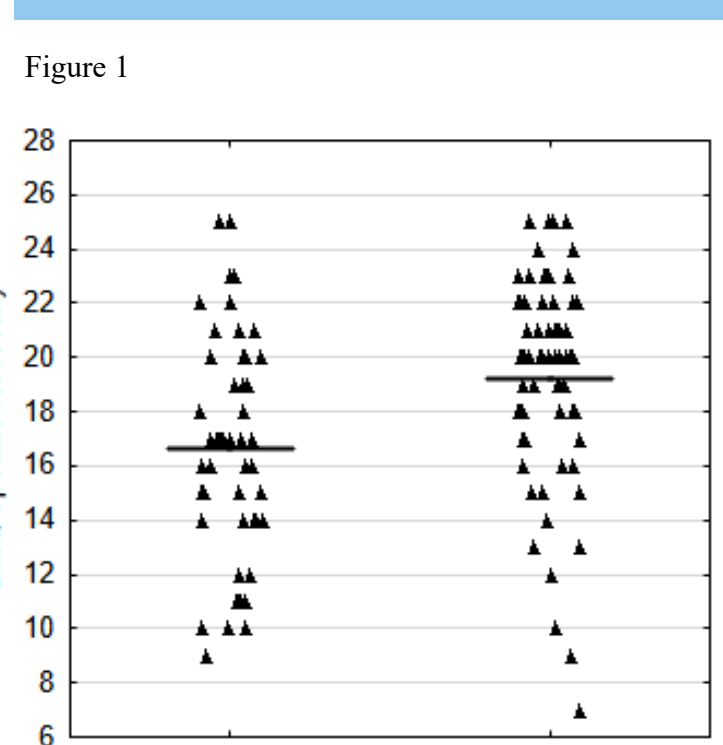

US
$\mathrm{POL}$

Country
- Mean (unadjusted) - Raw data
The study population was mostly females $(\mathrm{n}=96)$ and males $(\mathrm{n}=26)$ with a mean age $+/$ - SD of 68 between the two groups. The extent of genera beliefs that medicines are harmful and overused as well as the specific beliefs or concerns did not significantly differ between the studied populations $(\mathrm{p}>0.05)$. However, beliefs about the necessity of prescribed medications were significantly higher in the group of Polish $(n=69)$ patients as compared to the American ( $\mathrm{n}=50)$ (Figure 1) and the difference remained significant after adjusting for sociodemographic covariates $(18.3,95 \% \mathrm{Cl}$ 17.2-19.5 v. 16.2, 95\%CI 14.9-17.5, p=0.02). Necessity-minus-Concen beliefs (Figure 2) significantly predited self-reported medication -Concern also after adjusting for sociod also after adjusting for sociodemographic covariates ( $\mathrm{r}=0.41,95 \% \mathrm{CI} 0.25$ $0.55, \mathrm{p}<0.0001)$, with no significant difference existing between the studied groups $(\mathrm{p}=0.65)$ n 1 MEDICAL UNIVERSITY

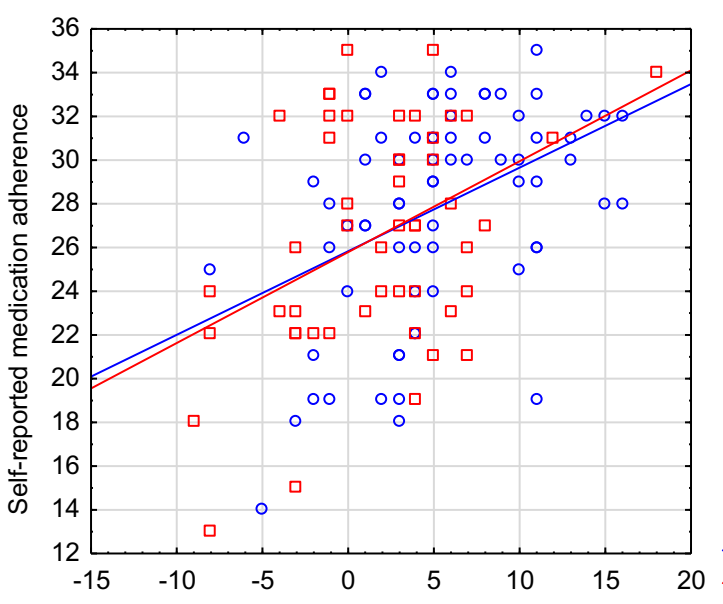

Q Country: POL

Figure 2

Necessity minus Concerns

\section{Conclusions}

Polish patients seem to believe that their cardiovascular medication are more necessary than American patients, however, the results may be biased due to the possible effect of unmeasured covariates and non- probabilistic sampling. The Necessity-Concerns Framework (NCF) appears equally useful in predicting self-reported adherence to cardiovascular medications in both populations. Calculated as Necessity of the medication subtracted from the Concerns about the treatment. treatment.

References

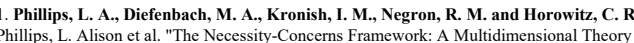

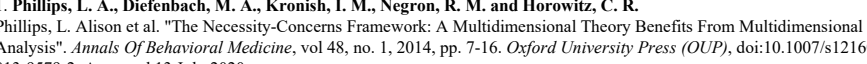

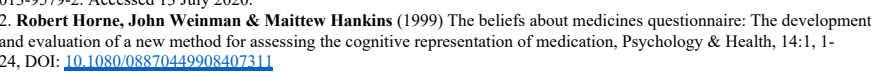

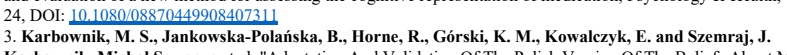

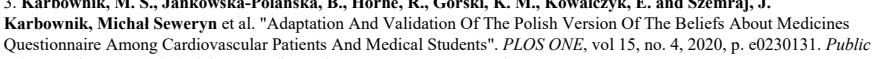

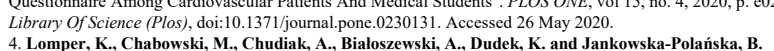

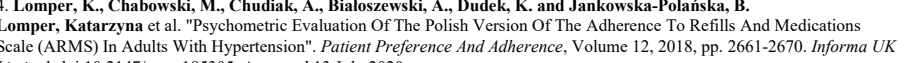

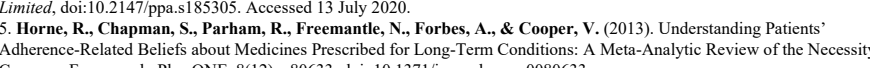

